\title{
Coaching and health
}

\section{John Launer}

In recent years, an increasing number of doctors have sought coaching or mentoring as part of their career development. Hospitals and other health organisations in the United Kingdom, the United States and elsewhere have set up schemes to provide these services to doctors, especially those who are starting in new jobs, or at points of transition in their working lives. In contrast to coaching in sports or academic subjects, coaching for doctors is not aimed at training in specific skills or giving advice. Instead, its purpose is to provide a time and space for reflecting on the challenges of medical work, including stress, time management, or the responsibilities of running a team. ${ }^{1}$ Ideally, coaches or mentors will have been trained to carry out the role, using techniques to facilitate reflection and decisionmaking, including non-directive questioning. (Although coaching and mentoring are sometimes defined in slightly different ways, in a medical context it makes sense to regard them as essentially the same). When these services are set up well and use properly trained coaches, there is evidence that they can have a significant effect on job satisfaction and professional effectiveness. ${ }^{2}$

As doctors have become more familiar with the principles and practice of coaching, and have experienced its benefits for themselves, they have inevitably started to consider how the same approach might be useful for many of their patients, such as those who are facing important dilemmas or turning points in their lives, or are having to manage long term medical conditions. There is of course nothing new about placing an emphasis on empowerment in patient encounters, and a number of other approaches also encourage this. These approaches include motivational interviewing, ${ }^{3}$ patient-centred medicine, ${ }^{4}$ shared decision-making ${ }^{5}$ and narrative-based medicine. ${ }^{6}$ However, what appears to be unique about applying coaching to the patient encounter is that it explicitly accords patients the same status and respect that one would offer to a professional colleague. This includes acknowledging that every adult is capable of finding and applying their own resources to address the difficulties they face in their lives, and the doctor's role is to facilitate this process, rather than telling people what to do, or simply giving them information in the hope that this alone will enable them to make informed choices.

Correspondence to Dr John Launer, Faculty Development, Health Education England, Stewart House, 32 Russell Square, London WC1B 5DN, UK; john.launer@nwl.hee.nhs.uk

\section{COACHING STANCE}

In a recent book called "Coaching for Health", two experienced practitioners and teachers of coaching, Jenny Rogers and Arti Maini, point out how well-suited a coaching stance is for medical practice in the twenty first century, where people have direct access to an enormous amount of medical information through the internet and other sources, and may no longer look to doctors as the sole fount of knowledge about their illnesses. ${ }^{7}$ They argue that a coaching stance also matches the needs of populations with a changing demography, where the bulk of medical workload consists of patients with complex, long term conditions who face the challenge of managing their symptoms and treatment from day to day, over many years or possibly decades. Most of all, the stance makes sense for helping people whose problems arise from lifestyle habits such as smoking or lack of exercise, and are unlikely to respond to straightforward advice or medical exhortation.

Rogers and Maini describe a wide range of techniques for engaging patients in a coaching relationship as responsible adults. They address such issues as how to balance empowerment with direct action where this is clinically essential (for example, in life threatening situations). They look at ways of building trust with patients who take a passive or fatalistic approach to their health and lifestyle. They also explain how factual information - such as the benefits and risks of different treatments - can be seamlessly interpolated into a coaching conversation, even in short consultations. Rogers and Maini question the assumption that coaching techniques are inapplicable to patients with learning difficultie or mental health problems, those from non-western cultures, or those who do not speak the same language as the doctor. They show how coaching can be adapted to overcome the challenges that these each present. They point out that coaching also reduces stress in practitioners, who are able to relinquish their ingrained sense of responsibility for fixing everyone else's problems. In sum, they make a compelling case that a coaching stance should be the default position for doctors in most patient encounters, unless there are compelling reasons such as a emergency to depart from it.

\section{POSITIVE RESULTS}

There has now been a considerable amount of research into the effect that coaching can have on patients. The results have been highly positive. For example, in a systematic review of health coaching in adult patients with chronic diseases, a team from Finland showed that it can be used to motivate patients to change their life style and improve their self care. ${ }^{8}$ They found statistically significant results in physiological, behavioural, psychological or social outcomes in 11 out of the 13 well-designed studies that they reviewed. These outcomes included weight loss, increased physical activity and reduced $\mathrm{HbA} 1 \mathrm{c}$ in diabetic patients. Other reviews have shown similar results, including sustained improvement over time. ${ }^{9} \quad$ Interestingly, a significant number of interventions that have been researched have been carried out not just by physicians but by psychologists, medical assistants and specially trained health coaches - something that will appeal to health service managers and economists because of its cost efficiency. Some of the interventions have even been carried out over the phone rather than face-to-face, adding further savings.

If health coaching was a new drug, scientists and commercial companies would be extolling its clear advantages and promoting its use. The fact that health coaching can be easily taught, readily applied, costs relatively little, has benefits for practitioners as well as patients, and appears to be free from harms, should lead us to consider introducing it into medical training and routine practice.

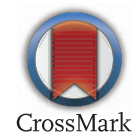

To cite Launer J. Postgrad Med J 2016;92:492.

Postgrad Med J 2016;92:492.

doi:10.1136/postgradmedj-2016-134356

\section{REFERENCES}

1 Garvey R, Stokes P, Megginson D. Coaching and Mentoring: Theory and Practice. London: Sage, 2008.

2 Chadwick-Coule T, Garvey B. London Deanery Mentoring Service: A Formative and Developmental Evaluation of Working Practices and Outcomes. Sheffield: Coaching and Mentoring Research Unit, Sheffield Business School, 2009

3 Stewart M, Brown JB, Weston W, McWhinney IR. Patient-Centred Medicine: Transforming the Clinical Method, $3^{\text {rd }}$ Edition. Abingdon: CRC Press, 2013.

4 Elwyn G, Frosch D, Joseph-Williams N, et al. Shared decision-making: a model for clinical practice. J Gen Intern Med 2012;27:1361-67.

5 Miller WR, Rollnick S. Motivational Interviewing: Helping People Change. New York NY: Guilford Press, 2013

6 Launer J. Why narrative? PMJ 2009;85:167-8.

7 Rogers J, Maini A. Coaching For Health: Why It Works and How To Do It. Maidenhead: Open University Press, 2016.

8 Kivelä K, Elo S, Kyngäs H, Kääriäinen M. The effects of health coaching on adult patients with chronic diseases: a systematic review. Patient Ed Couns 2014; 97:147-157.

9 Sharma AE, Willard-Grace R, Hessler D, et al. What happens after health coaching? Observational study 1 year following a randomized controlled trial. Ann Fam Med 2016;14:200-7. 